\title{
Erratum to: Estimating covariate functions associated to multivariate risks: a level set approach
}

\author{
Elena Di Bernardino ${ }^{1}$. Thomas Laloë ${ }^{2}$. \\ Rémi Servien $^{3}$
}

Published online: 22 May 2015

(C) Springer-Verlag Berlin Heidelberg 2015

\section{Erratum to: Metrika DOI 10.1007/s00184-014-0498-4}

Unfortunately, in the original publication, the value of $p_{n}$ in Theorem 4 was incorrectly published as

$$
p_{n}=o\left(v_{2, n}^{\frac{p}{p+1}} / T_{n}^{\frac{d+(d-1) p}{p+1}}\right)
$$

The correct value should read:

$$
p_{n}=o\left(v_{2, n}^{\frac{1}{p+1}} / T_{n}^{\frac{(d-1) p}{p+1}}\right)
$$

Also, in Example 2, the value of $v_{2, n}$ has been incorrectly published as:

$$
v_{2, n}=o(\sqrt{n})
$$

The correct value should read:

$$
v_{2, n}=o\left(\frac{n^{p / 2}}{T_{n}^{d}}\right)
$$

The online version of the original article can be found under doi:10.1007/s00184-014-0498-4.

Thomas Laloë

thomas.laloe@unice.fr

1 Conservatoire National des Arts et Métiers, IMATH, EA4629, 292 Rue Saint Martin, 75003 Paris, France

2 CNRS, LJAD, UMR 7351, Université de Nice Sophia-Antipolis, 06100 Nice, France

3 INRA, UMR1331 Toxalim, Research Centre in Food Toxicology, Universite de Toulouse, 31027 Toulouse, France 